\title{
AN UNUSUAL PRESENTATION OF KALA-AZAR IN BANGLADESH
}

\author{
AHMEDUL KABIR ${ }^{1}$, MD ROBED AMIN ${ }^{2}$ APARNA BISHWAS ${ }^{3}$, MOHAMMAD AL-AMIN ${ }^{4}$, SHEGUFTA MISHKET $^{2}$ \\ MUKERRAMA $^{5}$, SHANTA ROY ${ }^{6}$, SWADESH BARMAN $^{7}$
}

\begin{abstract}
:
Kala-azar is a vector borne zoonosis which is common in Mymensing, Tangail, and adjoining areas of Bangladesh. Usually patients have spleenomegaly during early presentation. Lymphadenopathy is also uncommon in this subcontinent. Moreover, most of the patients are rK39 positive. This case is reported because our patient had no spleenomegaly upto about 1.5 months after fever, and then developed mild spleenomegaly and cervical lymphadenopathy about 2 months later. Patient also was rK39 negative who finally diagnosed by FNAC from lymph node.
\end{abstract}

\section{Introduction:}

Leishmaniasis is a vector borne zoonosis having variable clinical presentations in the form of visceral, cutaneous (of localized or diffuse types) and mucocutaneous types depending upon the Leishmania species and immune responses of the hosts. Leishmaniasis threatens 350 million people in 88 countries across four continents and still remains a major public health problem in several underdeveloped countries ${ }^{1}$. Leishmanial infection does not lead to clinical disease in all cases as asymptomatic and subclinical forms are frequent. These infections may lead to subsequent active clinical disease when the immune status of the patient changes. The classical presentation of kala azar includes fever, asthenia, weight loss, anemia,splenomegaly, hepatomegaly and sometimes adenopathy. Splenomegaly appears early and increases gradually in relation to the duration of the disease ${ }^{1,2}$. Although there has been report of kala azar with lymphdenopathy in sudan and Chinese kala azar cases, rarely there has been reported of such incidence in Indian kala azar patients. The subclinical forms of the disease in the endemic areas remain undiagnosed and can become a clinical challenge to the treating physician. We present here a case report of a young man with unusual presentation of visceral leishmaniasis.

\section{Case report:}

A 32 years old male, by profession a service holder hailing from Potuakhali, Bangladesh presented to us on 19.01.2013 with the complaints of fever for one \& half months and loss of appetite, weight loss, generalized weakness \& occasional vomiting for the same duration. Fever was high grade, intermittent, was associated with chills \& rigor, subsided with sweating after taking antipyretic. The highest recorded temperature was $104^{\circ} \mathrm{F}$. There was no history of evening rise of temperature or night sweats. The fever was also associated with occasional vomiting which was non-projectile, not bile stained, scanty, contained food particles and did not contain blood. He also complained of anorexia, generalized weakness \& weight loss which is $5 \mathrm{kgs}$ within last one $\&$ half months. Patient gave no history of yellow coloration of sclera, urine or stool, abdominal distension, chest pain, respiratory distress or hemoptysis. He is non diabetic, non asthmatic \& gave no $\mathrm{H} / \mathrm{O}$ contact with $\mathrm{TB}$ pt. He has no history of travel to Kala-azar endemic zones. On admission he was ill looking with below average body build and nutritional status, mildly anaemic, generalized darkening of skin was present, absent bony tenderness, bilateral Inguinal lymphadenopathy were present, small, about $1.5 \mathrm{~cm}$ in size, firm in consistency, discreet, non-tender, mobile. Blood Pressure was $80 / 40 \mathrm{~mm}$ of $\mathrm{Hg}$ in lying $\& 60 / 30 \mathrm{~mm}$ $\mathrm{Hg}$ on standing. Temperature was $101^{\circ} \mathrm{F}$. Pulse 120 beats/min, regular with normal volume. Regarding systemic examination on admission his oral cavity was normal, Liver was moderately enlarged in size, about $5 \mathrm{~cm}$ from right costal margin along the mid clavicular line, surface was smooth with regular margin, consistency was firm, non-tender and moved with respiration. Upper border of liver dullness was in right $4^{\text {th }}$ intercostal space along right mid clavicular line. Hepatic bruit was absent. Spleen was not

1,2. Associate professor, Department of Medicine, Dhaka Medical College Hospital

3. Assistant professor, Department of Medicine, Dhaka Medical College Hospital

4. Registrar, Department of Medicine, Dhaka Medical College Hospital,

5,6,7. Honarary Medical Officer, Department of Medicine, Dhaka Medical College Hospital

Bangladesh J Medicine 2013; 24 : 35-37 
palpable. Other systemic examinations revealed no abnormality.

We investigated the patient and found his heamoglobin level $12 \mathrm{gm} / \mathrm{dl}$, ESR $20 \mathrm{~mm}$ in 1 st hour (Westergreen), Total WBC count 4,000/cu mm, differential count : Neutrophil 65\%,Lymphocytes 34\%, Platelet count 15,000/cu mm. On peripheral blood film there was thrombocytopenia with microcytic hypochromic blood picture. Urine $\mathrm{R} / \mathrm{E}$ : albumin $1+$,Blood urea $54 \mathrm{mg} / \mathrm{dl}$, Serum creatinine $2.20 \mathrm{mg} /$ dl, Serum electrolyte: Na $125 \mathrm{mmol} / \mathrm{L}, \mathrm{K} 3.5 \mathrm{mmol} /$ L , Cl $98 \mathrm{mmol} / \mathrm{L}$. After 7 days serum creatinine reduced to $1.8 \mathrm{mg} / \mathrm{dl}$. Blood culture report showed no growth. USG of whole abdomen showed bilateral slightly echogenic kidneys and right sided trace pleural effusion, S. ACTH: 29.80pg/ml (Normal: 8.3$57.8 \mathrm{pg} / \mathrm{ml}$ ), Basal Cortisol : $665 \mathrm{nmol} / \mathrm{L}$ (Basal range:116-1065nmol/L), Rapid ACTH Stimulation test: Cortisol 30min: 1649.90nmol/L, Cortisol 60min: $1859.90 \mathrm{nmol} / \mathrm{L}$, Bonemarrow study: Hypocellular marrow with maturation arrest of granulopoietic series at myelocyte stage, Chest $X$ ray, Serum Bilirubin , ECG were normal ,Serum Albumin 2.62 gm $\backslash \mathrm{dl}$, SGPT $66 \mathrm{U} \backslash \mathrm{L}$, Prothombin Time : pt: 28sec, control: 12sec, INR: 1.05, HBs Ag, Anti $\mathrm{HCV}$, HIV,Sputum for AFB, MT Test, ICT for Kala-azar (rK39 test), ICT for Malaria and ANA test were Negative. We examined the patient daily. On 28-01-13 we found bilateral cervical lymphadenopathy (Anterior cervical chain) which were small in size, 3 in number, largest one is about $2 \mathrm{~cm}$ in size, firm in consistency, nontender, mobile, discreet, no discharging sinus was present. On abdominal examination we found just palpable Spleen. Repeat USG of whole abdomen showed mildly enlarged liver, Parenchymal echotexture was uniform, Spleen was mildly enlarged in size, mainly in transeverse diameter $(5.3 \mathrm{~cm})$ and enlarged lymph nodes are noted around splenic hilum $(1.2 \mathrm{~cm})$, both adrenal glands are enlarged in size,

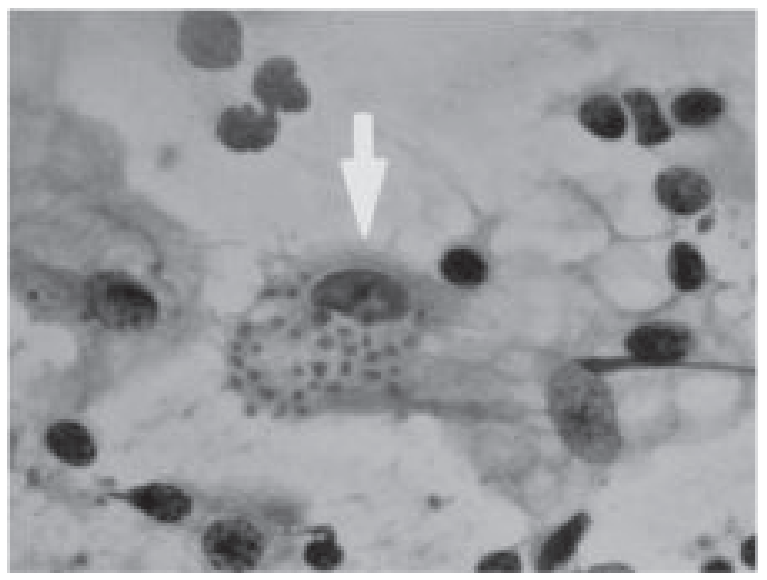

more in left with diffusely heterogenous parenchymal echotexture $(6.3 \times 3.1 \mathrm{~cm})$. Repeat CBC (after getting 3 units of whole blood and 3 units of platelet concentrate) showed $\mathrm{Hb} 6 \mathrm{gm} / \mathrm{dl}$, WBC 3200/cmm, Platelet $4000 / \mathrm{cmm}$. FNAC from Rt cervical lymphnode showed small lymphocytes \& histiocytes, these also reveal many Leishman donovan bodies. We started Injectable Liposomal Amphorericin B. Patient felt well first 2 days. However, his condition deteriorated from $3^{\text {rd }}$ day and ultimately he did not survive.

\section{Discussion:}

Leishmaniasis is a vector borne zoonosis with rodents and canids as common reservoir hosts and humans as incidental hosts. The two Leishmania species usually held responsible for visceral leishmanaiasis are $L$. donovani and $L$. infantum. Visceral leishmaniasis ranges over the intertropical zones of America and Africa, and extends into temperate regions of South America, Southern Europe and Asia. There are an estimated 12 million cases worldwide with one and a half to two million cases occurring each year. ${ }^{1} 90 \%$ of the visceral leishmaniasis cases in the world are in Bangladesh, India, Nepal, Sudan and Brazil. Population movements such as rural to suburban migration in Northeastern Brazil ${ }^{2}$ are factors for visceral leishmaniasis extension by exposing thousands of non immune individuals to the risk of infection.

Visceral leishmaniasis has variable clinical presentations in the form of visceral, cutaneous (of localized or diffuse types) and mucocutaneous types depending upon the species and immune response of the hosts. Visceral leishmanaiasis is a disease of the mononuclear phagocytic system, commonly affecting the spleen, liver, lymph nodes and bone marrow. But other organs (intestines, lung) and tissues (skin) may also become involved, as they contain the elements of mononuclear phagocytic system. In immunosuppressed individuals and in advanced cases, all body organs are involved.

The presence of amastigotes within macrophages remains the hallmark of Leishmania infection. It is a chronic disease, evolving slowly over many months or even a few years. Spontaneous evolution is fatal in $90 \%$ of cases, mainly caused by intercurrent infections or hemorrhages. The incubation period is difficult to evaluate precisely. It is generally two to six months, but can range from 10 days to many years. Long incubation periods, up to 10 years have been occasionally reported, ${ }^{3}$ related to clinical outcome of asymptomatic infection following immune system alteration. The onset of the disease may be sudden or gradual. In sudden onset there is rapid rise in temperature, gradual insidious onset includes irregular fever, slowly increasing, sometimes, with a transitory fall to sub febrile levels which can delay diagnosis. 
The classical presentation of kala-azar includes fever, asthenia, weight loss, anemia, splenomegaly, hepatomegaly and sometimes adenopathy. Splenomegaly appears early and is almost invariably present. Spleen size increases gradually in relation to the duration of the disease. Discrete superficial lymph nodes can appear during evolution. The nodes are small, firm painless and mobile. Peripheral adenopathy is more common in Sudan, and Indian kala azar is usually not accompanied by peripheral lymphadenopathy. Leishmanial infection does not lead to clinical disease in all cases; asymptomatic and subclinical forms are frequent. This has been demonstrated in various epidemiological surveys. ${ }^{4,5}$ Badro et al. ${ }^{2}$ characterize the subclinical form of visceral leishmaniasis as non specific mild clinical manifestations lasting for more than three weeks including fever, cough, diarrhea, malaise, mild hepatomegaly and eventually splenomegaly presenting as fluctuating course that evolves over a prolonged period of time.

Whether different clinical presentations including atypical ones are due to different stages of clinical evolution of a chronic disease or variations in the pathogenicity of the organism or the immune status of the host is open for debate. A cohort study was planned in Brazil from January 1998 to December 2000 on 784 children aged between $0-5$ years to study clinical-laboratory profile of sub clinical form of visceral leishmaniasis. ${ }^{6}$ This cohort study also demonstrated that the combination of fever, hepatomegaly, hypergammaglobulinemia and increased blood sedimentation rate can predict the subclinical form of visceral leishmaniasis provided it is not associated with splenomegaly or leucopenia. Whereas the study by Badaro et al. ${ }^{2}$ demonstrated that the subclinical form of the disease was more common (almost 60\% of patients testing positive for antibodies to Leishmania), this study from Brazil had only $17.4 \%$ of the patients as subclinical cases. The occurrence of splenomegaly and leucopenia distinguished the acute form from subclinical form.

Badro et al. ${ }^{2}$ did not find lymphadenopathy as one of a common clinical sign in the subclinical form of disease. Though lymphadenopathy along with splenomegaly has been characterized in kala azar; lymphadenopathy without splenomegaly is not very well known in the disease. This opens a question for debate that can "active" kala azar present without splenomegaly. Anemia is the major and most frequent hematological sign. Generally of normocytic and normochromic type, it progressively and regularly increases until becoming intense (can become as low as $4 \mathrm{gm} / \mathrm{dl})$.

Our report reinforces the recommendation that in endemic and even nonendemic areas the possibility of visceral leishmaniasis be considered in patients presenting with fever more than two weeks, where spleenomegaly may be absent initially. The present case report highlights a number of atypical presentations of kala azar.

Firstly, patient remained undiagnosed for a considerable period of time being in the subclinical time frame. Once the disease evolved into active disease due to a superadded infection, it had many atypical things attached to it. There were lymph nodes without splenomegaly, which is not very well heard of. Lymph nodes in Indian variety of kala azar are rare, but lymph nodes without splenomegaly have not been reported so far as an active disease. Our patient had no LD body in bone marrow. Although the patient had persistent pancytopenia. We had not find any LD body in bone marrow which indicates its limitation of sensitivity than that of spleen. As the spleen was not palpable initially, the splenic aspiration was not tried in this case. $\mathrm{Rk} 39$ was also negative which is also surprising finding in this case report. May be the subclinical form had not achieved adequate antibody level in the blood to make it positive - However, spleen became just palpable about 1.5 months later. Delayed diagnosis due to atypical manifestations can lead to fatal outcome in patients of kalaazar. Instead of relying solely on the classical clinical features of visceral leishmaniasis, simple laboratory findings like pancytopenia can help make a clue for searching early diagnosis even in atypical cases, thereby reducing the mortality of visceral leishmaniasis. The clinical temporal profile of patients is very crucial and development of new symptoms and signs are to be followed up regularly to elucidate the diagnostic confirmation. However, FNAC from lymph node can also demonstrate presence of Kala-azar where Rk39 dipstick test and aldehyde test is negative.

\section{References:}

1. Desjeux P. Global control and Leishmania HIV coinfection. Clin Dermatol 1999;17:317-25.

2. Badaro R, Jones TC, Carvalho EM, Sampaio D, Reed $\mathrm{SG}$, Barral A, et al. New perspectives on a subclinical form of visceral leishmaniasis. J Infect Dis 1986;154:1003-11.

3. WRIGHT MI. Kala-azar of unusual duration, associated with agammaglobulinaemia. Br Med J 1959;1:1218-32.

4. Pampiglione S, Manson-Bahr PE, La Placa M, Borgatti MA, Musumeci S. Studies in Mediterranean leishmaniasis. 3. The leishmanin skin test in kalaazar. Trans R Soc Trop Med Hyg 1975;69:60-8.

5. Carvalho EM, Barral A, Pedral-Sampaio D, Barral-Netto $\mathrm{M}$, Badaró R, Rocha $\mathrm{H}$, et al. Immunologic markers of clinical evolution in children recently infected with Leishmania donovani chagasi. J Infect Dis 1992;165:535-40.

6. Gama ME, Costa JM, Gomes CM, Corbett CE. Subclinical form of the American visceral leishmaniasis. Mem Inst Oswaldo Cruz 2004;99:889-93. 